\title{
Depolarization Near-field Scanning Optical Microscopy: Influence of Wavelength and Tip Shape on the Lateral Resolution
}

\author{
G. von Freymann, M. Wegener and Th. Schimmel* \\ Institut für Angewandte Physik, Universität Karlsruhe, D-76128 Karlsruhe, Germany
}

\begin{abstract}
We have demonstrated recently the operation of depolarization near-field scanning optical microscopy (DPNSOM), a technique that allows optical near-field imaging with uncoated optical fibre tips by making use of the depolarizing effect of the tip-sample near-field. We have shown both experimentally and by computer simulations that optical subwavelength resolution is obtained. Here, the dependence of the DP-NSOM imaging contrast and resolution on the wavelength and the tip shape is investigated in detail by computer simulations. We show explicitly that the resolution obtained does not depend on the wavelength of light, thus demonstrating true near-field imaging. The results show that the DP-NSOM imaging process does not depend critically on details of the shape of the fibre tip. Copyright (C) 1999 John Wiley \& Sons, Ltd.
\end{abstract}

KEYWORDS: optical near-field microscopy; internal reflection mode; depolarization; computer simulations; DP-NSOM; tip-shape; uncoated fibre tips

\section{INTRODUCTION}

Conventional near-field scanning optical microscopy (NSOM) allows optical imaging and spectroscopy with lateral resolutions well below the diffraction limit by using small subwavelength apertures for illumination and/or detection of light. Therefore, it not only depends critically on the quality and reproducibility of these apertures, but also suffers from the problem that the evanescent field coming out of subwavelength-sized apertures decreases exponentially with the width of the apertures and leads to very low light intensities and thus low signal-to-noise ratio and long sampling times for acquiring conventional NSOM images.

Depolarization near-field scanning optical microscopy (DP-NSOM) avoids both problems by not using the small apertures. Near-field resolution is achieved with uncoated fibre tips as follows. A linearly polarized electromagnetic wave is coupled into an optical fibre and arrives at a tip with a nanometre-scale apex. Such tips can be produced routinely by a melting/pulling process or by etching procedures. No metal coating is applied to this tip - in contrast to conventional NSOM. ${ }^{1,2}$ Obviously, much of the light intensity will leak out at the side walls of this fibre and possibly will be reflected back into the propagating mode of the fibre. As a consequence of this, much of the reflected light stems from the far field and standing wave patterns can occur. This light is not desired because it does not allow subwavelength resolution of the sample.

The basic idea of DP-NSOM is the elimination of this far-field contribution from the light coming from

* Correspondence to: Th. Schimmel, Institut für Angewandte Physik, Universität Karlsruhe, D-76128 Karlsruhe, Germany.

Email: Thomas.Schimmel@phys.uni-karlsruhe.de the near-field of the sample beneath the tip apex by making use of the depolarizing effect of the tip-sample near-field. For this reason, a second polarizer (P2 in Fig. 1) is introduced in front of the detector (D), which is cross-polarized to P1. Detection of the component that is polarized orthogonal to the incident wave defines the orthogonal polarization (OP) mode of detection. If we detect the component parallel to the incident polarization (IP), the IP mode is used.

Let us first consider the OP mode, which obviously suppresses the undesired light: small, subwavelengthsized objects, e.g. holes or edges, depolarize a linearly polarized electromagnetic wave. Consequently, the tip without any sample leads to a certain depolarization and thus to a finite signal in the OP mode of detection. In the same way, a small object under investigation that

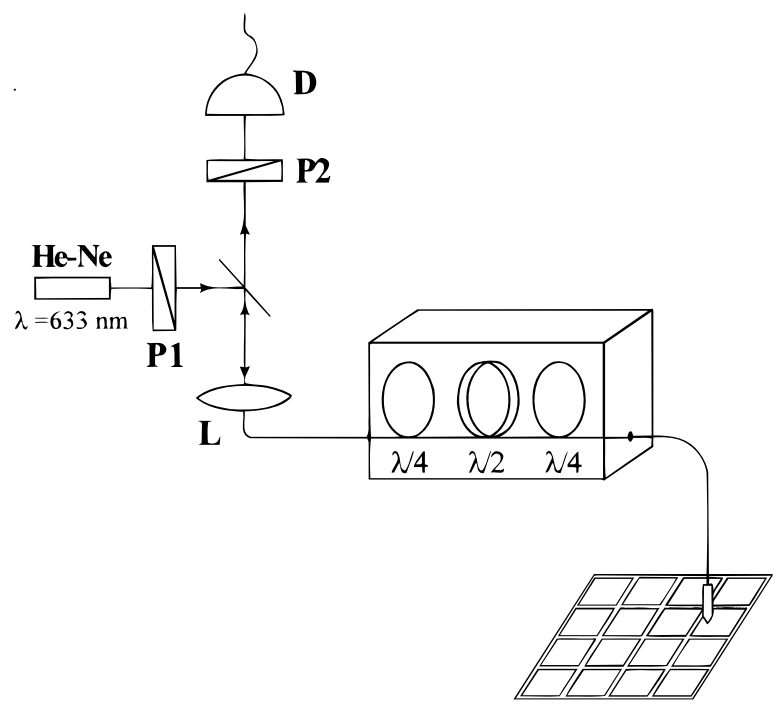

Figure 1. Schematic illustration of the experimental set-up. 
is separated from the tip leads to depolarization and hence to an OP mode signal. These two signals introduce a certain background that contains no information about the sample under investigation. If, however, the tip and the object are adjacent to each other, their combined depolarization is larger than the sum of the two backgrounds and thus more signal is detected in the OP mode. This combined depolarization gives the image contrast of the DP-NSOM. Computer simulations ${ }^{3}$ have confirmed this intuitive picture, leading to the following results on the DP-NSOM imaging:

(1) The DP-NSOM imaging is highly sensitive to dielectric changes perpendicular to the polarization of the incident light. This can be advantageous because it suppresses constant backgrounds that contain little information about the sample.

(2) A lateral resolution of $\sim 10 \%$ of the wavelength was demonstrated using a realistic tip shape.

(3) In contrast to the OP mode, the IP mode does not deliver optical near-field resolution.

(4) Scanning the fibre tip in the constant distance mode gives different images to those obtained by scanning in the constant height mode. This difference can be interpreted as an artefact of the constant distance mode: image contrasts are modified because, in this mode, the feedback loop changes the height of the tip. Consequently, we avoid the constant distance mode and always use the constant height mode throughout this article.

Although our theoretical work has demonstrated unambiguously optical subwavelength resolution with DP-NSOM, it has also shown one major experimental challenge: if, for some reason, the polarization of light before it enters the tip-sample region is slightly elliptical rather than linear, this leads to a mixing of the signals of the OP and the IP modes and, because the IP-mode signal is much stronger, no subwavelength resolution is possible any more. Unfortunately, even small bends or torsions of conventional optical fibres introduce a certain birefringence, which leads to elliptical polarization. Also, melted/pulled fibre tips often show temporal fluctuations of the polarization state. This is possibly due to built-in strain that remains after the melting, pulling and subsequent rapid cooling procedure, and slowly relaxes with time.

Our recent experimental work (Ch. Adelmann, J. Hetzler, G. Scheiber, Th. Schimmel, M. Wegener, H. B. Weber and $H$. von Löhneysen, unpublished results) has shown that these problems can be overcome by two steps. First, we use etched rather than pulled fibre tips, thus eliminating the problems with temporal fluctuations of the polarization state of light. Secondly, we introduced a polarization manipulator integrated within the fibre. This is merely a commercially available sequence of three mechanically adjustable fibre loops (equivalent to a quarter-wave plate, a half-wave plate and a second quarter-wave plate), which allows adjustment for linear polarization at the fibre tip, whatever the rest of the fibre does to the polarization. It also allows the axis of linear polarization to be rotated with respect to the sample. With this set-up (Fig. 1) and a well-defined test sample produced by electron beam lithography, we have been able to verify unambiguously three important points predicted by theory $(\mathrm{Ch}$. Adelmann, J. Hetzler, G. Scheiber, Th. Schimmel, M.
Wegener, H. B. Weber and H. von Löhneysen, unpublished results):

(1) In DP-NSOM imaging those edges that are perpendicular to the polarization of the incident light are imaged preferentially. When the incident polarization is parallel to the lines of a grid-pattern, we can either image the vertical or the horizontal lines. For $45^{\circ}$ polarization with respect to the grating, both vertical and horizontal lines are imaged simultaneously.

(2) A $150 \mathrm{~nm}$ resolution was obtained experimentally at a wavelength of $633 \mathrm{~nm}$. This corresponds to less than a quarter of the wavelength. As the tip is withdrawn from the sample, a near-field to far-field transition of the image contrast is observed, while the lateral resolution decreases. At heights above approximately half the wavelength of light, far-field images are obtained, resembling the image contrast of conventional light microscopes.

(3) Elliptical rather than linear polarization at the fibre tip leads to a deterioration of the lateral resolution, even for small ellipticities.

The DP-NSOM has three major advantages compared to conventional aperture NSOM:

(1) The signal levels obtained in DP-NSOM are much larger (by orders of magnitude), so that no lock-in technique is required. This, in principle, allows complete optical images to be measured within $1 \mathrm{~s}$ or even faster.

(2) The procedure for the production of the fibre tips does not require a metal coating. Consequently, the entire process is much simpler and more reproducible. Also, the tips are less sensitive to wear during scanning.

(3) Because no far-field illumination or far-field collection of light is involved (unlike conventional aperture NSOM), DP-NSOM is perfectly suited for implementation within a helium cryostat in a very compact fashion.

In this article we focus on two important questions:

(1) Is DP-NSOM imaging independent of the wavelength?

(2) Is the DP-NSOM imaging process stable in the sense that it does not depend critically on details of the shape of the fibre tip?

\section{COMPUTER SIMULATIONS}

\section{Theoretical methods and simulated geometries}

The method for numerical solution of the full set of vectorial Maxwell equations has been described previously. ${ }^{3-5}$ It implies computation of the electric field beginning from the nanometre-scale apex of the tip up to the end of the piece of fibre, the length corresponding to one wavelength of light. Integrating the Poynting vector-either for the OP mode or for the IP modeover a certain cone simulates the coupling of light into the propagating mode of the fibre and delivers exactly the signal that an experiment would measure in the configuration depicted in Fig. 1.

The three fibre tips used in the computer simulations below are shown in Fig. 2. The shape of the tip in Fig. 2(a) corresponds to the large opening angles that are 


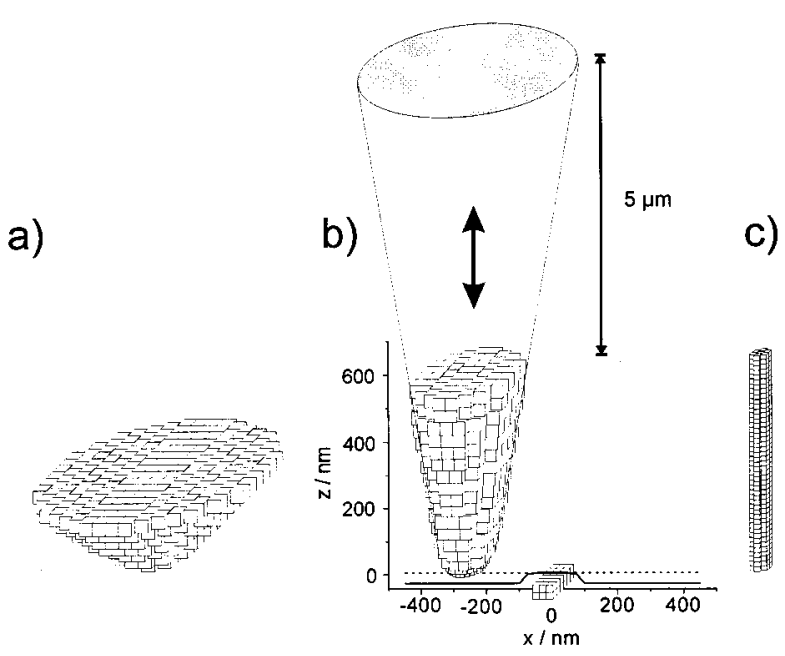

Figure 2. The three different fibre tips used in our computer simulations $(\mathrm{a}-\mathrm{c})$, together with the simulated geometry and the imaged rectangular glass sample (b).

typical for etched fibre tips (Ch. Adelmann, J. Hetzler, G. Scheiber, Th. Schimmel, M. Wegener, H. B. Weber and $\mathrm{H}$. von Löhneysen, unpublished results). The tip of Fig. 2(b) corresponds to the typical shape and opening angle of a fibre tip obtained by the usual pulling procedure. This shape has been used in the simulations presented in Ref. 3. Finally, Fig. 2(c) is a somewhat artificial tip shape that continues the trend from (a) to (b) towards smaller opening angles. This tip is merely of theoretical interest: it is useful for understanding but is difficult to fabricate experimentally.

\section{Wavelength dependence}

Figure 3 shows the dependence of the DP-NSOM (in OP mode) image on the wavelength of light for the tip shown in Fig. 2(b). The height of the tip is kept constant at a value of $5 \mathrm{~nm}$ above the sample surface. The object is a small, free-standing glass bar (width in scanning direction $=60 \mathrm{~nm}$, depth $=120 \mathrm{~nm}$, height $=40 \mathrm{~nm}$ ). Scanning is performed perpendicular to the long side of the bar and parallel to the incident polarization of light. Three relevant wavelengths are selected because they are accessible experimentally: $457 \mathrm{~nm}$ (one of the blue lines of an argon ion laser), $633 \mathrm{~nm}$ (the prominent red line of the helium-neon laser) and $1060 \mathrm{~nm}$ nearinfrared radiation (close to the neodymium-doped yttrium-aluminium-garnet (ND : YAG) laser). This corresponds to a variation in wavelength of more than a factor of two.

From Fig. 3(a) it is obvious that the features seen in the OP mode do not change qualitatively with wavelength. In particular, for all three wavelengths two peaks - one at each edge of the glass bar-are observed. Note that their positions do not change with wavelength, a behaviour that is expected for true near-field optical imaging. The contrast decreases with increasing wavelength, an effect that can be understood as follows: for decreasing object size at a fixed wavelength, it is obvious that the image contrast goes to zero. If, on the other hand, the wavelength is increased at a fixed object size, the effect is the same because only the ratio of object size and wavelength matters.


Figure 3. The DP-NSOM line scans for three different wavelengths of light both for the OP mode (a) and for the IP mode (b). Note that in the OP mode the shape of the signal and the exact position and distance of the maxima do not depend on the wavelength, consistent with true optical near-field resolution. This is in contrast to imaging in the IP mode, where the shape of the curves and the resolution depend on the wavelength. The images were obtained with fibre tip (b) (see Fig. 2).

On the other hand, the optical images change with wavelength in the IP mode of detection [Fig. 3(b)]. This is consistent with our discussion above and is explained by the fact that the light that leaks out at the edges of the fibre tip and is reflected back into the fibre does not deliver optical subwavelength resolution. Consistent with this interpretation, the signal levels are much larger in IP mode than in OP mode [compare scales on the axes in Figs 3(a) and 3(b)].

\section{Tip shape dependence}

In the following section, the influence of the tip shape on DP-NSOM imaging is investigated in the constant height mode. Figure 4 shows, the corresponding DP-NSOM line scans. Sample, polarization and scan direction are identical to those used in Fig. 3. The wavelength of light is $633 \mathrm{~nm}$. For each tip shape, the calculations are performed for three different scan heights.

It is obvious that the overall shape of the DP-NSOM signal is the same as discussed above: one maximum at each edge of the glass bar is observed. Few details change. The constant signal background is larger for a larger opening angle of the tip [Fig. 4(a)] than for a smaller opening angle [Fig. 4(b)]. This can be interpreted as being due to an increased contribution of the light that couples out of the side walls of the fibre, is 

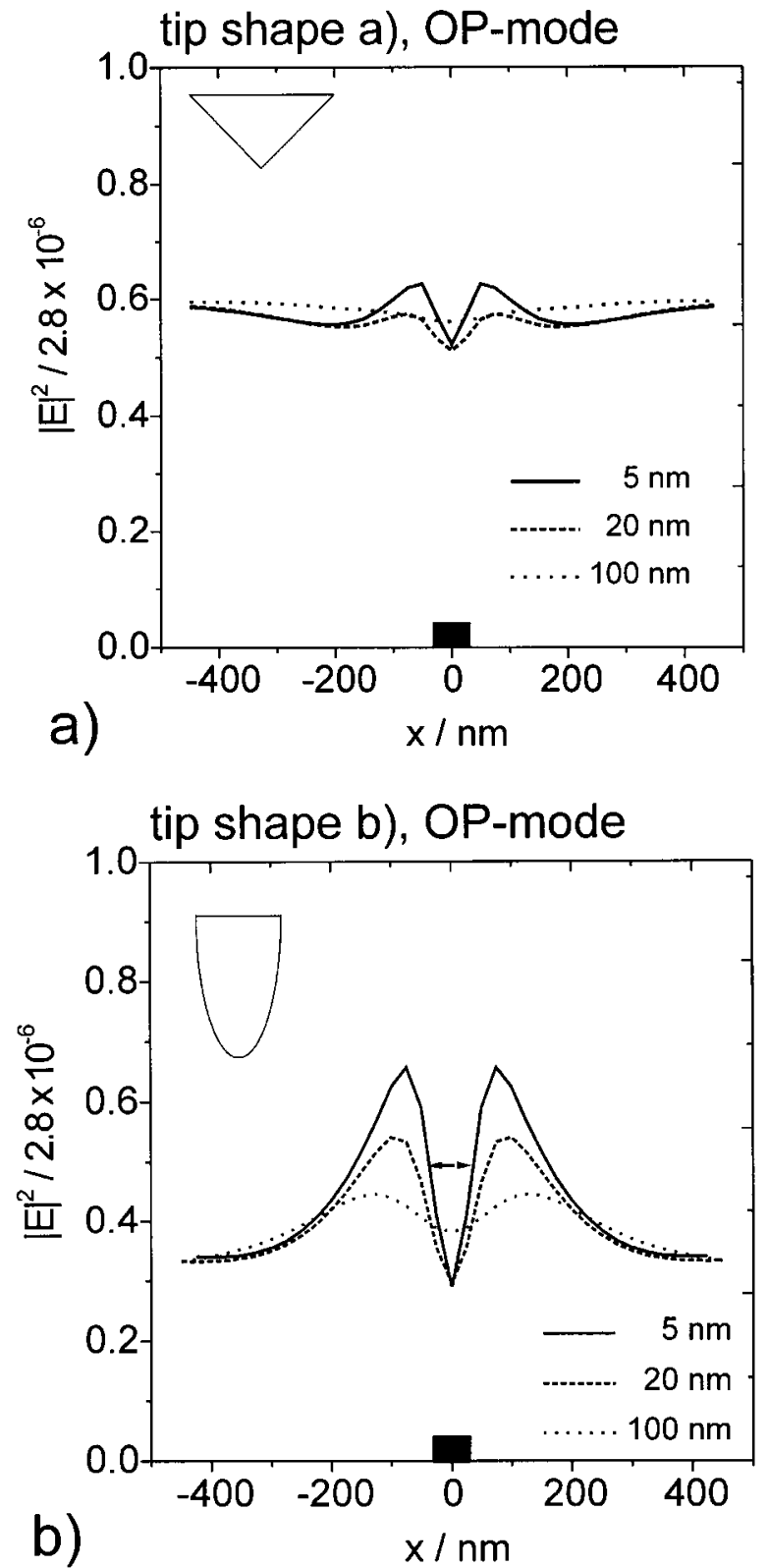

Figure 4. Tip shape dependence: DP-NSOM line scans obtained in the OP mode for the three different tip geometries shown in Fig. 2 (wavelength $633 \mathrm{~nm}$ ). The arrow in Fig. 4(b) corresponds to a full width at half-maximum of $71 \mathrm{~nm}$, obtained by scanning across a $60 \mathrm{~nm}$ wide glass sample with aperture (b).

reflected and depolarized on the sample and finds its way back into the fibre mode. The optical resolutions of the fibre tips of type (a) and type (b) are almost identical. Somewhat better optical resolution is obtained with the artificial tip shape of type (c). The constant signal background is almost gone and suppression of the undesired components of light is further improved. As

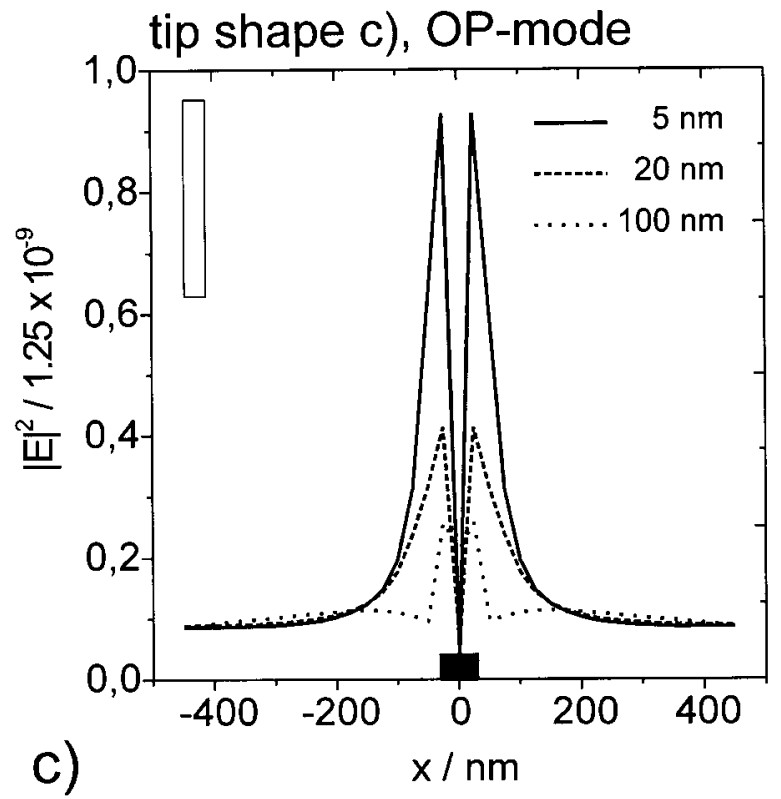

Fig. 4. Continued

pointed out above, such a tip cannot be produced in reality, but the calculations show how, in principle, optical resolution could be improved further, by optimizing tip design, e.g. by a combination of the tip shapes of type (a) and type (c).

The corresponding calculations that we performed in the IP mode (not shown in Fig. 4) show that, in contrast to the OP mode, subwavelength resolution is obtained neither for tip (a) nor for tip (b). This is consistent with the above discussion and explains the negative experimental results reported by other research groups. ${ }^{6}$

\section{CONCLUSION}

The influence of wavelength and tip shape on NSOM image contrast and resolution obtained with uncoated fibre tips was investigated by computer simulations. The results give further evidence that DP-NSOM delivers true optical near-field resolution. In particular, the results show that resolution in the OP mode does not depend on the wavelength of light and that the imaging contrast does not critically depend on details of the shape of the fibre tip.

\section{Acknowledgement}

This research has been supported by the DFG (Sonderforschungsbereich 195) and by the Karlsruhe Forschungsverbund Nanotechnologie-Funktionselemente.

\section{REFERENCES}

1. D. W. Pohl, W. Denk and M. Lanz, Appl. Phys. Lett. 44, 651 (1984).

2. E. Betzig, P. L. Finn and J. S. Weiner, Appl. Phys. Lett. 60 2484 (1992).

3. G. von Freymann, Th. Schimmel, M. Wegener, B. Hanewinkel, A. Knorr and S. W. Koch, Appl. Phys. Lett. 73, 1170 (1998).
4. O. J. F. Martin, C. Girard and A. Dereux, Phys. Rev. Lett. 74, 625 (1995)

5. G. von Freymann, Th. Schimmel and M. Wegener, Appl. Phys. A 66, $\mathrm{S939}$ (1998)

6. V. Sandoghar, S. Wegscheider, G. Krausch and J. Mlynek, J. Appl. Phys. 81, 2499 (1997). 